\title{
GIUSEPPE BOTTAI E IL RINOVAMENTO FASCISTA DELL'UNIVERSITÀ ITALIANA (1936-1942)
}

Por Luigiaurelio Pomante. Milano: Franco Angeli, 2018, 133 páginas. ISBN 97888-917-1493-0.

Giuseppe Bottai (1895-1959) es uno de los dirigentes del ventennio fascista italiano cuya trayectoria política ha suscitado mayores controversias. Como muchos de sus correligionarios, tuvo una precoz y fulgurante carrera política. Luchó como voluntario en la Gran Guerra, y en marzo de 1919 fue uno de los fundadores del fascio de Roma. Fascista, pues, de primerísima hora, resultó elegido diputado en las elecciones de 1921, aunque no pudo retener el acta pues — como también ocurrió con Dino Grandi o Roberto Farinacci, otros destacados líderes fascistas- no tenía la edad mínima para ejercer como diputado. Pese a su juventud, tuvo un papel muy destacado en los debates políticos internos del Partido Nacional Fascista (PNF) de los años veinte, así como en la elaboración (desde su cargo de subsecretario del Ministerio de las Corporaciones) de la Carta del Lavoro. En 1929 ya formaba parte del Gran Consejo del Fascismo, y ese mismo año fue nombrado ministro de las Corporaciones, cargo que desempeñó hasta 1932. Vendrían luego cargos de diversa entidad hasta su nombramiento como ministro de Educación Nacional en 1936, puesto en el que se mantuvo hasta febrero de 1943.

Bottai, licenciado en derecho, tenía una fuerte inclinación por el debate teórico, lo que le llevó a fundar y dirigir la revista Critica fascista (1923), una publicación fundamental para seguir los principales debates que tuvieron lugar en el seno del fascismo italiano en los años veinte y treinta. Entre las preocupaciones fundamentales de Bottai estuvieron muy pronto, además del corporativismo, la cultura y la educación, así como la cuestión de la juventud y su papel en el nuevo orden fascista. 
Pese a su activismo político (participó de forma destacada en la Marcha sobre Roma y, muchos años más tarde, se fue como voluntario a la guerra de Abisinia), en los duros debates políticos de la segunda mitad de los años veinte, se alineó contra las tesis «intransigentes» y más movimentistas que podía encarnar Farinacci, y defendió las posiciones que orientaban el régimen por la vía de la institucionalización y de la subordinación del Partido al Estado.

Esta posición política ha servido de base argumental para una revisión de la trayectoria de Bottai que tiende a presentarlo como una especie de fascista «amable», lejos de la violencia y el sectarismo que suele identificarse con el fascismo. Su «moderación» política y su interés por las cuestiones culturales y educativas permiten trazar una imagen de él muy alejada de la del fascista que impone su ley con el manganello y el aceite de ricino. A ello contribuyeron no poco sus dulcificadas memorias (Vent'anni e un giorno), publicadas en Milán en 1949. Y es que, como ocurrió con no pocos jerarcas fascistas, Bottai sobrevivió mejor que bien al fin del régimen. Pese a sus votos de fidelidad al Duce hasta el último momento, en la decisiva reunión del Gran Consejo del Fascismo del 25 de julio de 1943, se alineó con los que votaron la destitución de Mussolini. Siguieron años difíciles (en los que fue condenado a muerte -en ausencia- en el proceso de Verona, 1944, en plena República de Saló), pero de los que salió indemne. En 1947 pudo acogerse a la amnistía política y volver a Italia, reincorporarse a la universidad - había obtenido en 1931 una cátedra de derecho corporativo en la Universidad de Pisay vivir rehabilitado políticamente hasta su muerte en 1959.

La visión de Giuseppe Bottai como un fascista moderado, amable y heterodoxo, abierto al debate y respetuoso con la discrepancia política -incluso la de los antifascistas - es la que pudo leerse en dos biografías del político italiano publicadas a finales de los años setenta ${ }^{1}$ y que abrieron una dura discusión historiográfica en la que otros historiadores cuestionaron seriamente esa interpretación de su figura. ${ }^{2}$ Desde entonces, el debate no ha decaído, y se ha mantenido en la amplísima

\footnotetext{
1 G. Bruno Guerri, Giuseppe Bottai. Un fascista critico (Milán: Feltrinelli, 1976); Alexander J. De Grand, Bottai e la cultura fascista (Roma-Bari: Laterza), 1978.

2 Vito Zagarrio, «Bottai: un fascista critico?», Studi Storici 17, no. 4 (1976): 267-271; Emilio Gentile, «Bottai e il fascismo. Osservazioni per una biografia», Storia contemporanea X, no. 3 (1979): 551-570.
} 
bibliografía existente sobre fascismo, cultura e intelectuales y, no hay que decirlo, en las más recientes biografías del personaje. ${ }^{3}$

Valga esta extensa introducción para poder situar mejor el libro que aquí se reseña. Su autor, Luigiaurelio Pomante, investigador en la Università degli Studi di Macerata, es un reconocido especialista en historia de la universidad. En la presenta obra se ocupa del pensamiento y la obra de Giuseppe Bottai en relación con la universidad, y, más específicamente, de su proyecto de reforma integral del sistema universitario italiano para adecuarlo a las necesidades del nuevo Estado y de la nueva sociedad fascistas. Pomante, buen conocedor de la materia que trata, aborda su trabajo desde una perspectiva que sitúa la obra, como más adelante se justificará, entre aquellas que optan por ofrecer el perfil más favorable o, si se prefiere, el menos abrupto del dirigente fascista, privilegiando, por tanto, los aspectos de apertura y tolerancia ideológica que pueden detectarse en algunas de las iniciativas políticas que impulsó Bottai, y dejando en penumbra otros aspectos menos presentables. De todo ello, se hablará a continuación.

El libro es una obra breve, dividida en dos partes de extensión similar. En la primera, Pomante se ocupa de exponer las ideas y el proyecto de Bottai para la universidad, y su aplicación práctica; en la segunda, se reproducen ocho textos del jerarca fascista, la mayor parte de ellos discursos, que cubren un amplio espacio temporal: el primero data de 1928 y el último de 1941. Hay que agradecer la publicación de estos textos - cada uno de ellos estrechamente relacionado con alguna de las cuestiones que se han analizado en la primera parte- pues permiten conocer de primera mano la elaboración teórica de Bottai en relación con la universidad.

Hay que indicar que el autor no se propone realizar un análisis exhaustivo de la obra de Giuseppe Bottai como ministro, ni siquiera en su vertiente universitaria. De hecho, el contenido del libro no encaja del todo con el marco cronológico que se apunta en el título y que hace referencia (aunque no exacta) a los años en que Bottai estuvo al frente del Ministerio de Educación Nacional. Con pleno acierto, Pomante arranca su estudio centrándose en las propuestas sobre la renovación universitaria que desgranó

\footnotetext{
3 Monica Galfré, Giuseppe Bottai. Un intellettuale fascista (Firenze: Giunti, 2000).
} 
Bottai en un discurso de 1928, cuando era subsecretario de Estado en el Ministerio de las Corporaciones. Y es que, como se ha dicho anteriormente, las cuestiones culturales y educativas venían ocupándole $-\mathrm{y}$ preocupándole- desde mucho antes de convertirse en el máximo responsable de la política educativa italiana. Bottai y los colaboradores de su revista Critica fascista participaron muy activamente - y de forma muy crítica- en los debates que tuvieron lugar en torno a la reforma educativa impulsada por Giovanni Gentile como primer ministro de Instrucción Pública en un gobierno presidido por Mussolini. A Bottai le preocuaba no solo - como a otros críticos de la reforma Gentile- el grado de auténtica fascistización de la escuela italiana (que muchos dentro del partido consideraban insuficiente), sino también problemas de más calado como la adecuación del modelo escolar (y ahí, como señala Pomante, Bottai incluía el conjunto del sistema educativo, incluyendo la universidad) a las necesidades de la nueva sociedad fascista y de una economía en acelerado proceso de modernización y que pretendía organizarse sobre bases corporativas. Igualmente le preocupaba - y podría decirse que de manera especial - la educación política de los jóvenes y la capacidad del sistema educativo para formar con ellos la nueva clase dirigente que debería, en un futuro no muy lejano, sustituir a la generación de la Marcha sobre Roma.

El autor, como decía, renuncia a un análisis sistemático de la obra de Giuseppe Bottai como ministro para centrarse en el análisis de algunas iniciativas concretas que aquel impulsó (antes de ser ministro y durante su mandato como tal) y que pretendían llevar a la práctica lo que, en buena medida, había expuesto en el citado discurso de 1928, que puede leerse íntegro en la segunda parte del libro.

En el capítulo 1 de la obra, el autor analiza el punto de partida de la reflexión de Bottai sobre la cuestión universitaria. Para Bottai, la cultura debía constituir una parte fundamental de la acción política del fascismo. El Estado debía impulsar la elaboración de una cultura propiamente fascista y en esa labor estaban llamadas a desempeñar un papel especial las universidades, que, sin embargo, debían pasar por un proceso previo de enérgica renovación. Bottai consideraba que el sistema universitario italiano estaba anticuado, poco adaptado a las nuevas necesidades políticas, sociales y económicas, y desconectado de la onda de cambio que había supuesto la «revolución» fascista. 
Para impulsar el cambio necesario, ya como ministro, Bottai promovió algunas iniciativas que son objeto de análisis por parte de Pomante. Por ejemplo (capítulo 2), la elaboración de una colección de monografías sobre la historia de cada una de las universidades italianas. El objetivo fundamental de esta iniciativa era poner en marcha un proceso de reflexión interna en todas las universidades, con participación de buen número de profesores, que se centraría no solo en la reconstrucción de su pasado, sino también en el debate sobre su función en el presente y en el futuro de Italia. Esta gran movilización debía culminar al cabo de unos años, con las monografías ya completadas, en la celebración de un gran congreso internacional sobre historia de la universidad italiana que supondría el impulso definitivo para la renovación que Bottai consideraba indispensable. El comienzo de la guerra en Europa y el alineamiento de Italia con el III Reich hicieron que el congreso se celebrase en Bolonia en abril de 1940 sin el alcance internacional que el ministro había previsto. Fue el «Primo Convegno nazionale per la storia delle università italiane», que Pomante analiza en el cuarto capítulo del libro.

Entre la puesta en marcha de la primera de estas iniciativas y la celebración del congreso de 1940, el ministro Bottai impulsó la aprobación de la Carta della Scuola (1939), o lo que es lo mismo, la que debía ser la auténtica reforma fascista de todo el sistema educativo. A la presencia de la universidad en la Carta dedica Pomante el tercer capítulo del libro. La bibliografía sobre esta cuestión es muy extensa y, en ese sentido, poco aporta de nuevo esta obra. Más interés tiene el análisis que en el mismo capítulo se hace de la puesta en marcha de una revista - también iniciativa del ministro-, Gli Annali della Università d'Italia. Rivista bimestrale dell'Istruzione Superiore, que debía fungir como agente impulsor del debate y de la reforma universitarios. En el primer número de la revista, el propio Bottai publicó un texto en el que sintetizaba su visión de la universidad y las funciones que a esta le cabía cumplir. Muy oportunamente, ese es uno de los textos que pueden leerse en la segunda parte del libro. Entre otras cuestiones relevantes, Bottai se ocupaba de problemas candentes de la universidad del momento como el de la masificación estudiantil (más percibida que real), el desempleo de los titulados universitarios, la inadecuación de las universidades a las necesidades del presente, etc. Y ponía el acento también en la necesidad de conectar el Estado con la ciencia que se hacía en las universidades y, muy especialmente, en el papel fundamental que 
tenía la universidad en la formación de la futura clase dirigente italiana, para lo cual consideraba imprescindible hacer más exigente el proceso de selección de los estudiantes, que, eso sí, debía regirse exclusivamente por criterios de mérito y capacidad.

La primera parte del libro concluye con un capítulo (quinto) dedicado a la revista Primato, lettere e arti d'Italia, fundada por Bottai en 1940, y más concretamente con el análisis del amplio debate sobre la universidad que tuvo lugar en sus páginas a lo largo de ocho números -entre febrero y junio de 1941-, en el que participó un gran número de intelectuales y de profesores, y que se cerró con un artículo del ministro en el que este realizó una síntesis de la discusión y fijó su diagnóstico sobre lo que quedaba por hacer. En realidad, como ha sido casi unánimemente señalado por la literatura - y esta obra no constituye una excepción-, la ambiciosa reforma que suponía la Carta della Scuola quedó en nada por el inicio de la guerra y, después, por el fin del propio régimen fascista. La universidad no escapó a ese destino.

Como ya se ha indicado anteriormente, estamos ante una obra breve que, por ello mismo, difícilmente puede dar cuenta de todo lo que su título promete. Consciente de ello, Pomante opta por centrar su estudio en algunas cuestiones concretas, dejando de lado otras que, sin embargo, no son menos relevantes para comprender la labor política de Giuseppe Bottai como ministro de Educación Nacional. Y esa selección es la que permite presentar el lado más amable del dirigente fascista y obviar los aspectos más reprobables de su actuación como ministro.

A la obra —otra vez el problema de la extensión - le falta contexto. A un lector poco familiarizado con las políticas educativas y con la situación de la universidad italiana durante el ventennio fascista le resultará difícil apreciar lo que de novedad suponían los planteamientos de Bottai. No hay ninguna referencia a la reforma del ministro Gentile, ni a los debates que suscitó ni a los sucesivos retoques a que se vio sometida por todos los ministros que le sucedieron hasta llegar a Bottai. Sin embargo, esa cuestión es capital para entender la insatisfacción de este con la situación en que se encontraba la universidad italiana a mediados de los años treinta. Uno de esos debates tenía que ver con el compromiso del profesorado con el régimen fascista y con la «solución» ideada por Gentile (no siendo ya ministro), y asumida por Mussolini, de someter a todos 
los profesores a un juramento de fidelidad al régimen. La posición de Bottai en ese debate, que era, en definitiva, una discusión sobre la libertad de cátedra y sus límites, no se estudia en el libro.

Al menos otras dos cuestiones capitales están ausentes en el análisis de Pomante. La primera, fundamental, es la responsabilidad de Giuseppe Bottai, en tanto que ministro, en la contundente depuración del profesorado universitario por motivos «raciales». Es decir, la expulsión de decenas de profesores y estudiantes judíos de las universidades italianas a partir de 1938. Nada se explica tampoco sobre su posición en el debate sobre ciencia y raza que precedió a la aprobación de las leyes raciales y su aplicación en la universidad.

El segundo tema relevante que está ausente del libro es el de los littoriali y el progresivo cierre de espacios de debate crítico en los mismos, que coincide precisamente con la etapa ministerial de Giuseppe Bottai. Hubiera resultado de interés un análisis de esa cuestión, pues no deja de resultar paradójico que se limitara la libertad de crítica en estos encuentros de estudiantes universitarios justo cuando al frente del ministerio estaba alguien que había hecho bandera de la defensa de ese derecho por parte de los jóvenes universitarios, precisamente como parte de su formación como futura clase dirigente del régimen.

Estas dos cuestiones, y muy especialmente la relativa a la depuración del profesorado y del estudiantado judío, casan mal con la imagen de un dirigente fascista moderado y tolerante, capaz incluso de aceptar la participación en una revista como Primato de autores que tenían planteamientos críticos con el fascismo o que, incluso, estaban ya en las filas del antifascismo. No se trata, quede claro, de que el autor pretenda «blanquear» la figura de Bottai; su condición de fascista no se oculta ni se relativiza en ningún momento. Sin embargo, en ese debate sobre su figura al que me refería al principio, Pomante prefiere realzar aquellos planteamientos y actuaciones de Bottai que refuerzan su imagen de dirigente moderado, ni que sea por oposición al fascismo que se suele identificar con la violencia más descarnada. En realidad, su vinculación con el mundo de la cultura no llevó nunca a Bottai a la denuncia de la violencia escuadrista; jamás se desdijo de su crítica a la democracia y el liberalismo ni renunció a su concepción corporativa de la sociedad y el Estado. Nunca fue un disidente del régimen ni mostró la más mínima duda 
sobre la superioridad —y la necesidad histórica— del fascismo. Si fue un moderado, lo fue en términos relativos, por comparación con auténticos energúmenos que abundaban en las jerarquías del partido y el Estado. Si defendió la libertad de crítica (dentro de un orden, tampoco hay que exagerar), siempre lo hizo partiendo de la premisa de que esa crítica solo era aceptable desde dentro del sistema y con la finalidad de perfeccionarlo. Bottai era, sencillamente, un fascista inteligente, pero eso no lo convertía necesariamente en un fascista crítico o en un político en la frontera con la tradición liberal.

El libro de Luigiaurelio Pomante es, en definitiva, una interesante aportación al conocimiento de la teoría y la praxis de Giuseppe Bottai en relación con la cuestión universitaria durante el ventennio fascista. Si en algunos aspectos no realiza aportaciones novedosas, en otros sí sugiere nuevas lecturas y abre caminos para futuros trabajos. Los textos que se reproducen en la segunda parte del volumen resultan útiles, como ya quedó dicho, y son un perfecto complemento del análisis que se lleva a cabo en la primera parte del libro. Al final, el lector se queda con las ganas de saber más, de que el autor hubiese ampliado el foco sobre cuestiones también relevantes para su objeto de estudio, sobre el que demuestra ser un buen especialista.

Francisco Morente Universitat Autònoma de Barcelona Francisco.Morente@uab.cat 